\title{
Thermodynamics of the quantum Ising model in the two-dimensional kagome lattice
}

\author{
Chyh-Hong Chern* and Mitsuaki Tsukamoto \\ Institute for Solid State Physics, University of Tokyo, \\ 5-1-5 Kashiwanoha, Kashiwa, Chiba 277-8581, Japan
}

\begin{abstract}
In the classical Ising model on the kagome lattice, there are macroscopically degenerate classical states with exponentially-decayed spin correlation. As the singular quantum perturbation is introduced by applying the transverse magnetic field, the quantum ground state remains disordered, dubbed by "disorder-by-disorder". Here we compute the temperature dependence of the spin susceptibility and the specific heat for difference strength of the field to understand the effect of the singular quantum perturbation in this system. The relevance to the $\mathrm{ZnCu}_{3}(\mathrm{OH})_{6} \mathrm{Cl}_{2}$ will be also commented.
\end{abstract}

PACS numbers: $75.30 . \mathrm{Gw}, 75.40 . \mathrm{Cx}, 75.40 . \mathrm{Mg}$

Macroscopically degenerate states often occur in the classical level in the anti-ferromagnetic systems that the exchange energy can not be perfectly shared by all the nearest neighbor spins. It happens naturally if spins are placed in the triangular geometry in the two-dimensional lattices. In two dimensions, triangles can share the edges to form the triangular network or share the corners to form the kagome lattice. If we consider the classical Ising dynamics in these two systems, both of them show macroscopically degenerate classical ground states. However, the spin correlation in these two systems are very different, which determines the fate of the consequent quantum ground states when the quantum dynamics is introduced. When the transverse magnetic field is introduced by the following Hamiltonian,

$$
H=J \sum_{<i j>} S_{i}^{z} S_{j}^{z}-\Gamma \sum_{i} S_{i}^{x}
$$

where $S^{k}=\sigma^{k} / 2$ and $\sigma^{k}$ are the Pauli spin matrices and $J$ is taken to be positive, the quantum ground state in the triangular lattice favors an spin-ordered ground state, the maximally-flippable state, because the spin correlation is critical in the classical model. On the other hand, the quantum ground state in the kagome case remains disordered because the spin correlation is exponentiallydecayed [1, 2].

In this paper, we focus on the kagome case and compute its thermodynamic properties with various $\Gamma$. At $\Gamma=0$, the spin susceptibility shows dramatic upturn in the low temperature and diverges at the zero temperature. For non-zero $\Gamma$, we also see the significant upturn but it saturates at the zero temperature. Our calculation is the first results on the thermodynamic quantities to distinguish the classical disordered state and the quantum disorder states. Furthermore, as the macroscopically degenerate classical states lead to the residual entropy at $T=0$, the quantum dynamics which lifts the degeneracy results in additional peak in the specific heat in the low temperature at the order of $\Gamma$. Due to these properties, our model might be relevant to the recent discovered $\mathrm{ZnCu}_{3}(\mathrm{OH})_{6} \mathrm{Cl}_{2}$, which shows the ab- normal upturn in the spin susceptibility and saturates at $T=0$. There have been several theoretical papers trying to describe this unusual property. A straightforward interpretation is the presence of the impurity [3] that contributes additionally to the susceptibility. However, a naive inclusion of the contribution from the impurity can explain the data only above $20 \mathrm{~K}[4]$. Different angles of views by considering the contribution from the spin anisotropy and spin-orbital interaction can somewhat show the upturn but can not show the saturation at $T=0$ due to the "minus-sign" problem in their calculation [5, 6]. Our calculation advances their results because there is no "minus-sign" problem in our model. Moreover, our phenomenological approach to understand the magnetic systems also opens a new way to explore the low-temperature properties that can never be reached by the present quantum Monte Carlo technique due to the technical difficulty.

In the following, we use the quantum Monte Carlo technique to compute the thermodynamical quantities of Eq.(1) by using the Trotter-Suzuki approximation [7]. To calculate the magnetic susceptibility, we have to include the perturbed Hamiltonian $\delta H=-h \sum_{i} S_{i}^{z}-h_{y} \sum_{i} S_{i}^{y}$. Discreting the imaginary time direction by $n$ steps, the $\mathrm{n}^{\text {th }}$ Trotter-Suziki approximant of the partition function is given by

$$
\begin{aligned}
& Z^{(n)}=\sum_{\left\{\sigma_{j k}\right\}} e^{-H_{\mathrm{eff}}^{\prime}}\left[\cosh \frac{\beta R}{2 n}\right]^{N n}, \\
& H_{\mathrm{eff}}^{\prime}=\frac{\beta J}{4 n} \sum_{k,<i j>} \sigma_{i k} \sigma_{j k}-\frac{\beta h}{2 n} \sum_{j, k} \sigma_{j k} \\
& -\frac{1}{2} \log \operatorname{coth} \frac{\beta R}{2 n} \sum_{j, k}\left(\sigma_{j k} \sigma_{j, k+1}-1\right)
\end{aligned}
$$

where $R^{2}=\Gamma^{2}+h_{y}^{2}, N$ is the number of the lattice sites, $\sigma_{j k}$ are now the classical variables taking only +1 and -1 , and the summation is over the $n$ stacks of the kagome lattices. The cluster algorithm is applied along the imaginary time direction. The thermodynamical quantities can be obtained by taking the derivatives on Eq.(2) with 
respect to the corresponding thermodynamical variables. The results are summarized as the following:

$$
\begin{aligned}
C v^{(n)} T^{2}= & \frac{1}{N}<\left(\frac{\partial H_{\mathrm{eff}}^{\prime}}{\partial \beta}\right)^{2}-\frac{\partial^{2} H_{\mathrm{eff}}^{\prime}}{\partial \beta^{2}}>-\frac{1}{N}<\frac{\partial H_{\mathrm{eff}}^{\prime}}{\partial \beta}>^{2} \\
& +\frac{R^{2}}{n \cosh ^{2} \frac{\beta R}{n}} \\
\chi_{z z}^{(n)}= & \frac{T}{N}\left(<\left(\frac{\partial H_{\mathrm{eff}}^{\prime}}{\partial h}\right)^{2}>-<\frac{\partial H_{\mathrm{eff}}^{\prime}}{\partial h}>^{2}\right) \\
\chi_{y y}^{(n)}= & \frac{T}{N}\left(<\left(\frac{\partial H_{\mathrm{eff}}^{\prime}}{\partial h_{y}}\right)^{2}-\frac{\partial^{2} H_{\mathrm{eff}}^{\prime}}{\partial h_{y}^{2}}>-<\frac{\partial H_{\mathrm{eff}}^{\prime}}{\partial h_{y}}>^{2}\right) \\
& +\frac{h_{y}^{2}}{n T R^{2} \cosh ^{2} \frac{\beta R}{n}}+\frac{\Gamma^{2}}{R^{3}} \tanh \frac{\beta R}{n} \\
\chi_{x x}^{(n)}= & \frac{T}{N}\left(<\left(\frac{\partial H_{\mathrm{eff}}^{\prime}}{\partial \Gamma}\right)^{2}-\frac{\partial^{2} H_{\mathrm{eff}}^{\prime}}{\partial \Gamma^{2}}>-<\frac{\partial H_{\mathrm{eff}}^{\prime}}{\partial \Gamma}>^{2}\right) \\
& +\frac{1}{n T \cosh ^{2} \frac{\beta R}{n}}
\end{aligned}
$$

where $\chi_{z z}, \chi_{y y}$, and $\chi_{x x}$ are defined by $d m_{z} / d h$, $d m_{y} / d h_{y}$ and $d m_{x} / d \Gamma$ respectively, and $m_{x, y, z}$ are the magnetization per site. For a fixed temperature, the leading term of the error between the Trotter-Suzuki approximant of the thermodynamical quantities and those obtained from Eq.(1) can be shown to be linear in $1 / n$. Therefore, our results are the extrapolation of the straight line at $n \rightarrow \infty$. For each $n$ and an ensemble, $10^{6}$ Monte Carlo Sweeps are taken. For each set of parameters, 64 ensemble averages are used.

Fig.(1) and (2) are the temperature dependence of the inverse of the longitudinal susceptibility $\chi_{z z}$ per spin for various $\Gamma$ and different sizes of the system. $L$ in the figures is the length of the linear dimension so that the number of total spins $N=3 L^{2}$. The period boundary condition is applied in both directions. The temperature is in the unit of $\mathrm{J}$ and the Boltzman constant is set to be 1. In high temperature, all curves are fitted well by the Curie-Weiss law regardless $\Gamma$ and $L$. As the temperature goes down, the $\chi_{z z}$ have the abnormal upturn and saturate at the values depending on the $\Gamma$ at $T=0$. Spin flipping helps reduce the upturn of the $\chi_{z z}$. Since our Monte Carlo results for $L=2$ is the same as the ones obtained by the exact diagonalization, we only report the exact diagonalization results for $L=2$ here. For $\Gamma=0$, the coupling constant in the imaginary-time direction is divergent so that we report the result of $L=2$ by the exact diagonalization only. At finite $\Gamma$, the ground state is quantum disorder. $L=4$ looks fairly enough for the calculation and the size dependence becomes weaker for smaller $\Gamma$. Moreover, since the ground state of the $\Gamma=0$ is known as a classical disorder state [1], we believe that the result of $L=2$ for $\Gamma=0$ is representative. Fig.(2) is the magnifying plot around the origin of the Fig.(1). It can be seen clearly that the $\chi_{z z}$ of the $\Gamma=0$ (classical disorder) diverges at $T=0$, while the ones at the finite

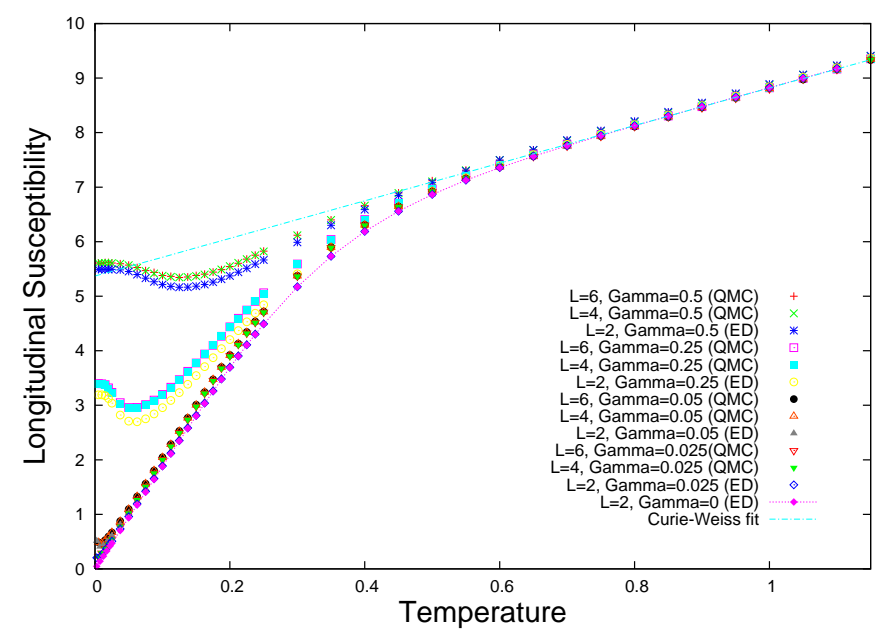

FIG. 1: (Color Online)The temperature (in the unit of $J$ ) dependence of the inverse of the $\chi_{z z}$ per spin. $\mathrm{L}=2,4,6$ correspond to $\mathrm{N}=12,48,108$ respectively. The blue straight line is the Curie-Weiss fit. QMC is denoted for the result from quantum Monte Carlo and ED is the one from exact diagonalization

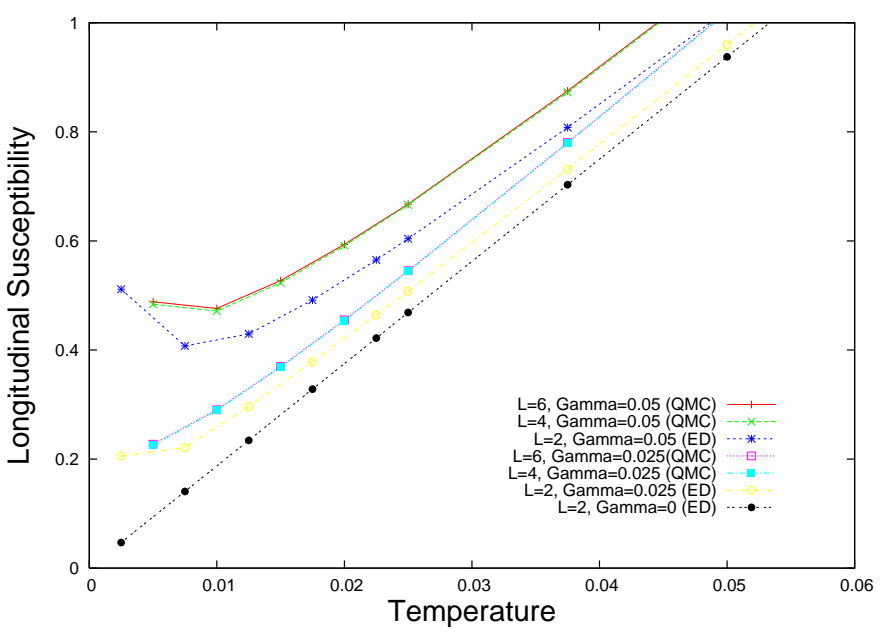

FIG. 2: (Color Online)The magnifying plot around the origin of Fig.(1). At $\Gamma=0$ (classical disorder), the longitudinal susceptibility diverges at $T=0$. At finite $\Gamma$ (quantum disorder) , they saturate at finite values at $T=0$. (Temperature is in the unit of $J$ )

$\Gamma$ (quantum disorder) saturate at $T=0$. This is the first result of the magnetic susceptibility thus far that distinguishes the quantum disorder from the classical disorder.

Experiments measured the magnetic susceptibility in the powder samples. In Fig.(3) and (4), we report the temperature dependence of the inverse of the powder susceptibility $\chi$ defined by $\chi=1 / 3\left(\chi_{x x}+\chi_{y y}+\chi_{z z}\right)$. One apparent feature is that the size scaling becomes even weaker. It is probably because $\chi$ is an averaged quantity. Because of the anisotropy, $\chi_{x x}, \chi_{y y}$, and $\chi_{z z}$ are different from one other. Surprisingly, $\chi_{y y}$ is larger than the others and the size scaling is small in $\chi_{y y}$. On the other 


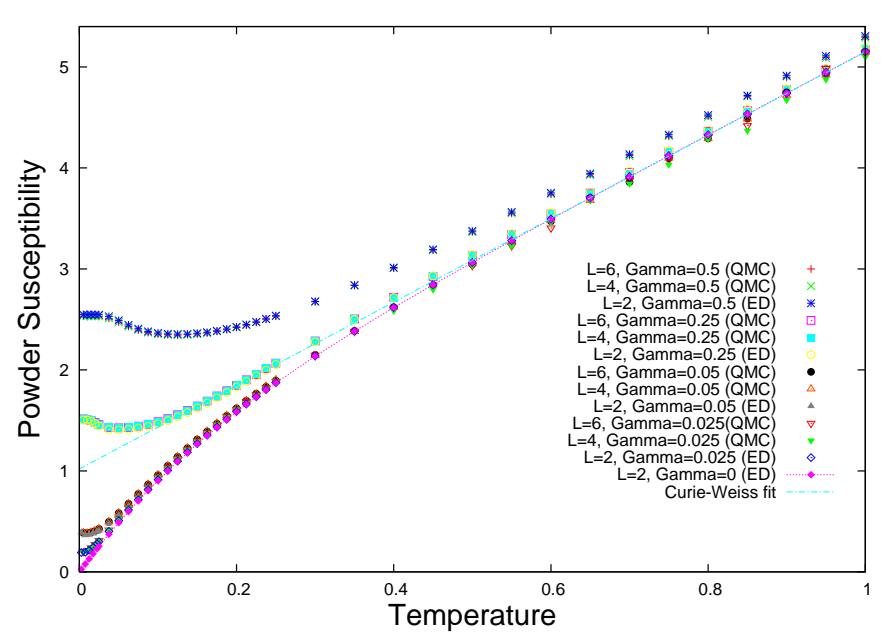

FIG. 3: (Color Online)The temperature dependence of the inverse of the powder susceptibility $\chi$ per spin. $\mathrm{L}=2,4,6$ correspond to $\mathrm{N}=12,48,108$ respectively. The blue straight line is the Curie-Weiss fit. QMC is denoted for the result from quantum Monte Carlo, and ED is the one from exact diagonalization. (Temperature is in the unit of $J$.)

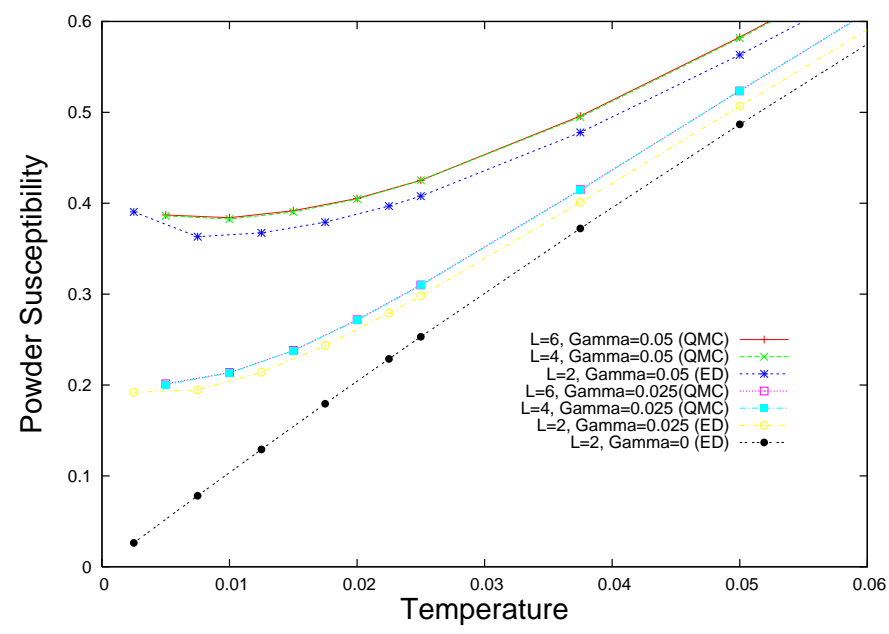

FIG. 4: (Color Online)The magnifying plot around the origin of Fig.(3). At $\Gamma=0$ (classical disorder), the powder susceptibility diverges at $T=0$. At finite $\Gamma$ (quantum disorder), they saturate at finite values at $T=0$. (Temperature is in the unit of $J$ )

hand, $\chi_{y y}$ and $\chi_{x x}$ become equal at $\Gamma=0$, which is consistent with expectation. Furthermore, the $\chi$ shows the upturn in the low temperature and saturates at the zero temperature for the small $\Gamma$ cases. In Fig.(4), the difference between classical disorder and the quantum disorder is again clearly seen. The $\chi$ of the quantum disorder saturates at $T=0$, and the one of the classical disorder diverges.

In Fig.(15), we report the temperature dependence of the specific heat with various $\Gamma$. At $\Gamma=0$, a broad peak locating at the order of $J$ is clearly observed. In this case, there is also a $\delta$-function peak at $T=0$ represent-

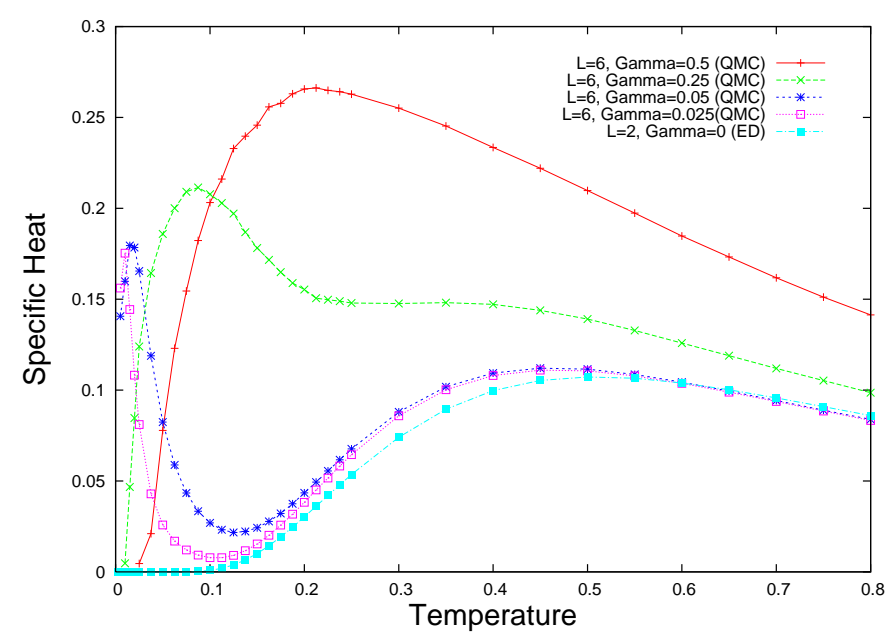

FIG. 5: (Color online)The temperature (in the unit of $J$ ) dependence of specific heat per spin. $\mathrm{L}=6$ corresponds to $\mathrm{N}=108$. QMC is denoted for the result from quantum Monte Carlo, and ED is the one from exact diagonalization

ing the macroscopic degeneracy of the classical ground states. At finite $\Gamma$, the transverse field lifts the degeneracy and therefore results in an additional peak locating at the order of $\Gamma$. For larger $\Gamma$, like 0.25 and 0.5 , two peaks emerges and a spin gap can be clearly seen. For $\Gamma=0.025$ and 0.05 , two peaks are well separated. The one at the order of $J$ at these two $\Gamma$ s are overlapping with the one at $\Gamma=0$. This indicates that the quantum perturbation has the dramatic effect on the ground states but not the higher-energy states. Unfortunately, our numerical resolution is not able to identify whether or not it is gapped or gapless for small but non-vanishing $\Gamma$. We remark that the states in the degenerate manifold at $\Gamma=0$ are connected by the single spin flip, i.e. gapless. Whether or not the gaplessness survives with respect to the quantum perturbation and extends to finite $\Gamma$ requires more detail study.

For the application to $\mathrm{ZnCu}_{3}(\mathrm{OH})_{6} \mathrm{Cl}_{2}$, there is only one parameter in our model to fit with the experiments. Although the $\Gamma$ term is understood as the external transverse magnetic field, it can also be regarded as the internal quantum spin flipping matrix. In addition, at a first glance, considering the Ising dynamics in the spin- $1 / 2$ system might be absurd for most of the experts. However, it has been shown that even if the spin is isotropic in the bulk of $\mathrm{Fe}, \mathrm{Ni}$, and $\mathrm{V}$, the effective spin interaction becomes anisotropic when the dimension is reduced, namely thin film, as long as there is non-vanishing spin-orbital interaction [8]. Therefore, that the spin in the spin- $1 / 2$ system is isotropic in 3 dimensions does not guarantee in 2 dimensions. In the presence framework, the saturation seen in the experiments [9, 10] can be easily understood. Namely, the quantum spin flip process dominates the low-energy physics as long as the Ising dynamics is 
relevant.

Our proposal can be tested experimentally by measuring the magnetic susceptibility on the single crystal sample of $\mathrm{ZnCu}_{3}(\mathrm{OH})_{6} \mathrm{Cl}_{2}$. If the spin flip process is relevant, experiments should see the shift of the saturation as the transverse magnetic field varies. Because the spin flip dynamics is enhanced, we expect the saturation shifts down as the field increases. In the very recent manuscript [11], this effect is observed in the powder sample. The applied magnetic field has two components: one is parallel to the hard plane and the other is longitudinal to the easy axis. Because the susceptibility is not sensitive to the longitudinal field, the decreasing saturation value with increasing applied field can be understood as the enhancement of the spin flipping process by the transverse field.

In summary, we compute the temperature dependence of the spin susceptibility and the specific heat of the quantum Ising model. Different from the Heisenberg model in the kagome lattice, the spin susceptibility in our model has the intrinsic upturn and saturation at finite value. In the specific heat, we observe the two-peak structure and the existence of the spin gap for larger $\Gamma$. The finite temperature properties in $\mathrm{ZnCu}_{3}(\mathrm{OH})_{6} \mathrm{Cl}_{2}$ indicates that the spin anisotropy and spin-flipping process might be dominant in the low temperature. In this case, the robust spin dynamics persists to the zero temperature might be the reason that $\mathrm{ZnCu}_{3}(\mathrm{OH})_{6} \mathrm{Cl}_{2}$ remains disordered at the $\mathrm{mK}$ range. In addition, there is no minus-sign problem in our model even though kagome is a highly frustrated system, which advances other methods technically to study the low-energy properties of the
$\mathrm{ZnCu}_{3}(\mathrm{OH})_{6} \mathrm{Cl}_{2}$.

CHC is indebted to Naoto Nagaosa and Masaki Oshikawa for very fruitful discussion. Part of the calculation is done by using the facilities in the Supercomputer Center in the Institute for Solid State Physics.

* Electronic address: chern@issp.u-tokyo.ac.jp

[1] R. Moessner, S. L. Sondhi, and P. Chandra, Phys. Rev. Lett. 84, 4457 (2000).

[2] R. Moessner and S. L. Sondhi, Phys. Rev. B 63, 224401 (2001).

[3] Y. Ran, M. Hermele, P. A. Lee, and X.-G. Wen, Phys. Rev. Lett. 98, 117205 (2007).

[4] G. Misguich and P. Sindzingre, unpublished, arXiv:07041017.

[5] M. Rigol and R. R. P. Singh, Phys. Rev. Lett. 98, 207204 (2007).

[6] M. Rigol and R. R. P. Singh, Phys. Rev. B 76, 184403 (2007).

[7] M. Suzuki, Progress of Theoretical Physics 56, 1454 (1976).

[8] J. G. Gay and R. Richter, Phys. Rev. Lett. 56, 2728 (1986).

[9] T. Imai, E. Nytko, B. Bartlett, M. Shores, and D. Nocera, unpublished, cond-mat/0703141.

[10] O. Ofer, A. Keren, E. Nytko, B. Bartlett, M. Shores, D. Nocera, C. Baines, and A. Amato, unpublished, cond-mat/0610540.

[11] F. Bert, S. Nakamae, F. Ladieu, D. L'Hote, P. Bonville, F. Duc, J.-C. Trombe, and P. Mendels, unpublished, arXiv:0710.0451 